\title{
Clinical Comparison of Laparoendoscopic Single-site Surgery and Multiport Laparoscopic Surgery for the Removal of Ovarian Endometrioid Cyst
}

\author{
Hongxia Yu ${ }^{\dagger}$, Zhiyong Dong ${ }^{\dagger}$, Wendi Zhang ${ }^{\dagger}$, Shoufeng Zhang, Jia Lu, Zhenyue Qin, Mingyue Bao, \\ Huihui Wang, Ying Cao, Yilin Sun, Wenjia Liu*, Jiming Chen* \\ Department of Gynecology, the Affiliated Changzhou NO. 2 People's Hospital of Nanjing Medical University, Changzhou, China
}

Email address:

810992072@qq.com (Wenjia Liu), cjming@126.com (Jiming Chen)

${ }^{*}$ Corresponding author

$\dagger$ Hongxia Yu, Zhiyong Dong and Wendi Zhang are co-first authors.

\section{To cite this article:}

Hongxia Yu, Zhiyong Dong, Wendi Zhang, Shoufeng Zhang, Jia Lu, Zhenyue Qin, Mingyue Bao, Huihui Wang, Ying Cao, Yilin Sun, Wenjia Liu, Jiming Chen. Clinical Comparison of Laparoendoscopic Single-site Surgery and Multiport Laparoscopic Surgery for the Removal of Ovarian Endometrioid Cyst. Journal of Gynecology and Obstetrics. Vol. 9, No. 2, 2021, pp. 46-49. doi: 10.11648/j.jgo.20210902.14

Received: March 8, 2021; Accepted: March 22, 2021; Published: March 30, 2021

\begin{abstract}
Background: Endometriosis is a common, chronic gynecological disease. Laparoscopy is currently the preferred method of ovarian endometriosis. However, the safety and feasibility of laparoendoscopic single-site surgery for ovarian endometrioid cyst removal still need to be discussed. Objective: To explore the safety and feasibility of laparoendoscopic single-site (LESS) surgery versus conventional multiport laparoscopic (CMPL) surgery for the ovarian endometrioid cystectomy. Methods: We retrospectively analyzed the data of 47 patients who had undergone LESS and LESS ovarian cystectomy due to ovarian endometrioid cyst in our hospital from March 2018 to April 2019. The patients were classified into single-port group (14) and multiport group (33) based on surgical paths. The patients' general characteristics and perioperative outcomes compared. Results: There were no significant statistical differences between the two groups $(\mathrm{P}>0.05)$ in the operation time and the maximum body temperature in $24 \mathrm{~h}$ after operation. There were significant statistical differences (all $\mathrm{P}<0.05$ ) in postoperative decrease of $\mathrm{Hb}$, intraoperative bleeding volume, total hospital stay and CS score. Conclusion: LESS for ovarian endometrioid cyst removal is safe and feasible, and it better than CMPL in relieving postoperative pain and inproving cosmetic effects and so on.
\end{abstract}

Keywords: Laparoendoscopic Single-Site Surgery, Ovarian Cystectomy, Ovarian Endometrioid Cyst

\section{Introduction}

Endometriosis (EMS) is a condition caused by the presence of functioning endometrial tissue (glands and stroma) outside the uterine cavity. EMS can affect all parts of the body, and the ovary is the most common site of invasion [1]. EMS invaded one or both ovaries and formed ovarian endometriosis, which mainly manifested as dysmenorrhea, infertility and pelvic mass, which seriously affected the quality of life of patients [2]. Laparoscopy is currently the preferred method of ovarian endometriosis as a minimally invasive procedure, but traditional laparoscopy still leave 3-4 scars on the abdominal wall. In recent years, with the improvement of laparoscopy skills, the more minimally invasive laparoendoscopic single-site (LESS) surgery has become more and more popular among patients, especially young women $[4,5]$. Laparoscopy is currently the preferred method of ovarian endometriosis [3]. From March 2018 to April 2019, 14 patients with ovarian endometrioid cyst were treated with laparoendoscopic single-site (LESS) surgery, and the results were satisfactory, compared with 33 patients with multiport laparoscopy surgery (MPLS), the safety and feasibility of the operation were evaluated.

\section{Information and Methods}

\subsection{General Information}

47 patients with ovarian endometrioid cyst who were treated in gynecology department of the Second People's 
Hospital of Changzhou affiliated to the Nanjing Medical University were investigated from March 2018 to April 2019, Inclusion criteria: (1) benign tumor of ovary. (2) the age of the patient was $21 \sim 53$ years, (3) the diameter of the ovarian cyst was less than $10 \mathrm{~cm}$. Exclusion Criteria: (1) malignant tumor of ovary. We included 14 patients with ovarian endometrioid cyst (single-port group) and matched 33 patients with ovarian endometrioid cyst (multiport group) according to the inclusion criteria and exclusion criteria.

\subsection{Method}

\subsubsection{Preoperative Preparation}

The preoperative examination was completed in both groups, and the contraindication of laparoscopy was eliminated. Preoperative gynecological examination to determine the activity of ovarian cysts, pelvic adhesion assessment. Routine preoperative preparation, cleaning of the umbilicus, bowel preparation, catheterization, monitoring of vital signs, preparation of routine laparoscopy instruments, preoperative placement of shoulder pads, intraoperative lying and head down and feet up.

\subsubsection{Surgical Procedure}

Under general anesthesia in LESS group, patients were put on their backs and heads down and feet in high position. After disinfection and towel spreading, intrauterine devices (except those without sexual life history) were placed. The skin, subcutaneous tissue, fascia and peritoneum were cut by $2.0 \mathrm{~cm}$ single incision in the direction of umbilicus, and the protective ring of 4.0-5.0 cm incision was placed, Triport cannula was attached and pneumoperitoneum tube was connected to establish artificial pneumoperitoneum. $10.0 \mathrm{~mm} 30^{\circ}$ laparoscopic lens and conventional laparoscopic instruments were inserted. The procedure of removing ovarian cyst is the same as that of laparoscopy. The ovarian cyst was removed and the wound of the ovary was sutured to stop bleeding. The ovarian cysts were removed completely and completely through the approach. Finally, the umbilical incision was sutured layer by layer with absorbable threads.

In the MPLS Group, a $10.0 \mathrm{~mm}$ longitudinal incision was made along the lower edge of the umbilical wheel, an artificial pneumoperitoneum was established by Verres needle puncture, $10.0 \mathrm{~mm}$ Trocar and laparoscopic lens were inserted successively, and $5.0 \mathrm{~mm}$ incision was made on the left and right lower abdomen and pubic symphysis respectively under the monitoring, $5.0 \mathrm{~mm}$ Trocar. After removal of the ovarian cyst, the wound of the ovary was sutured to stop bleeding and the specimen was removed through the umbilical incision. Suture the umbilical incision with the same method. The remaining incisions were sutured with only 3-0 silk.

\subsubsection{Outcome Measures}

The decrease of hemoglobin (HB), the operation time, the amount of blood loss, the postoperative complications, the total length of hospital stay, and the CS score.

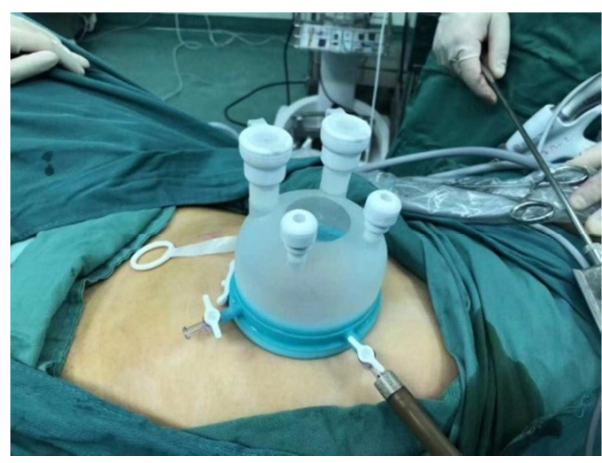

Figure 1. Constructing an operationchannel using a Triport sleeve.

\subsubsection{Statistical Methods}

The data were processed by SPSS 18.0 software. The results of measurement data of normal distribution were expressed by mean \pm standard deviation, and $\mathrm{T}$ test was used for comparison between groups, chi-square test was used for comparison of counting data, and rank test was used for non-parametric test. The difference was statistically significant with $\mathrm{P}<0.05$.

\section{Result}

\subsection{Comparison of General Clinical Data Between Two Groups}

The general clinical data of single-port group and multiport group were compared, including mean age [(30.4 \pm 7.0$)$, (29.3 \pm 5.0$)$ years], BMI $/(\mathrm{kg} / \mathrm{m} 2)$, abdominal operation history (LESS Group 5, MPLS Group 15), there was no significant statistical differences $(p=0.998,0.582>0.05)$. See Table 1 .

Table 1. Comparison of general data between two groups.

\begin{tabular}{llll}
\hline Group & age/age & BMI $/ \mathbf{k g} / \mathbf{m}^{\mathbf{2}}$ & History of previous abdominal surgery (cases) \\
\hline LESS Group $(\mathrm{N}=14)$ & $33.64 \pm 7.397$ & $23.72 \pm 2.93$ & 5 \\
MPLS group $(\mathrm{N}=33)$ & $36.64 \pm 7.254$ & $23.12 \pm 3.61$ & 15 \\
P value & 0.998 & 0.582 & $>0.05$ \\
\hline
\end{tabular}

\subsection{Comparison of Surgical Related Indexes Between the Two Groups}

The operation was successful in both groups, no conversion to laparotomy, and no additional hole was found in LESS group. Complications occurred in 1 case in LESS group, 1 case in small intestine injury, and 1 case in ureter injury in MPLS group $(\mathrm{P}>0.05)$. The operation time of LESS group $(129 \pm 49) \mathrm{min}$, MPLS group (105 \pm 38$) \mathrm{min}$, had no statistical significances $(p=0.077)$. The estimated blood loss in LESS group was more than MPLS group, and the decrease of hemoglobin was more significant ( $p$ value was $0.023,0.017$ ). See Table 2. 
Table 2. Comparison of perioperative related indexes between the two groups.

\begin{tabular}{llllll}
\hline Group & $\begin{array}{l}\text { Operation } \\
\text { Time/min }\end{array}$ & $\begin{array}{l}\text { Maximum body } \\
\text { temperature at 24 h after } \\
\text { operation }\end{array}$ & $\begin{array}{l}\text { Postoperative } \\
\text { stay/d }\end{array}$ & $\begin{array}{l}\text { Intraoperative bleeding } \\
\text { volume/ml }\end{array}$ & $\begin{array}{l}\text { Decrease of postperative } \\
\text { hemoglobin /(g/L) }\end{array}$ \\
\hline LESS Group (N=14) & $129 \pm 49$ & $37.4 \pm 0.3$ & $5.86 \pm 1.16$ & $57.50 \pm 41.91$ & $18.86 \pm 10.77$ \\
MPLS group (N=33) & $105 \pm 38$ & $37.3 \pm 0.3$ & $7.48 \pm 1.46$ & $27.58 \pm 24.21$ & $11.03 \pm 9.47$ \\
P value & 0.077 & 0.651 & 0.001 & 0.023 & 0.017 \\
\hline
\end{tabular}

\subsection{Comparison of Postoperative Indexes Between Two Groups}

There was no significant statistical differences in the highest body temperature $(\mathrm{p}=0.651)$ between the two groups. The length of hospital stay after operation was significantly different ( $\mathrm{p}$ value was 0.001 ). See Table 2. CS score was (22.5 \pm 2.6$)$ in single-port group and (17.2 \pm 2.3$)$ in multiport group, the difference was statistically significant $(\mathrm{p}<0.05)$. The aesthetic satisfaction of single-port group was significantly higher than that of multiport group.

\section{Discussion}

Endometrioid cysts of the ovary are the most common type of endometriosis. As a hormone-dependent disease, endometrioid cysts of the ovary are more common in young women and can involve one or both ovaries. Recurrence after surgery or drug therapy is a major characteristic of ovarian endometrioid cysts. In view of the minimally invasive and aesthetically pleasing nature of the laparoscopy, laparoscopy is currently the first choice for the treatment of ovarian endometrioid cysts and is suitable for the removal of cysts in the vast majority of patients [6].

Traditional laparoscopic ovarian cyst excision usually uses 3-4 puncture holes and leaves 3-4 scars on the abdominal wall. With the development of laparoscopic techniques and surgical instruments, clinicians are committed to reducing the number and length of surgical incisions in order to achieve postoperative scarless abdominal wall. Therefore, laparoendoscopic single-site (LESS) surgery came into being. The new procedure, LESS, is more popular with younger patients because younger women are more demanding and demanding [7]. The incidence of ovarian endometrioid cysts is higher in young patients, LESS seems to be more promising in the removal of ovarian endometrioid cysts.

Compared with traditional laparoscopy, LESS for ovarian cyst excision has obvious advantages: rapid postoperative recovery, short hospital stay, and no scarring of umbilical incision [8-10]. But because LESS is a procedure in which the laparoscope enters the field almost parallel to the instrument, it lacks the traditional three or four hole laparoscopy. LESS has the so-called "Chopstick Effect", which is more difficult to perform than conventional laparoscopy and requires more surgical equipment and skills from the operator $[11,12]$. In this study, LESS group had more intraoperative bleeding than MPLS group, and the hemoglobin decreased significantly before and after operation $(\mathrm{p}<0.05)$. Compared with traditional laparoscopy, LESS is not suitable for all patients because of its narrow visual angle, which can not find the bleeding point in time, or because of the difficulty of operating angle, which can not stop bleeding quickly and timely.

Ovarian endometrioid cysts often adhere to the pelvic wall, posterior broad ligament, uterosacral ligament, pelvic floor peritoneum and intestine, which leads to the changes of pelvic floor anatomy [13]. Therefore, the operation of ovarian endometrioid cyst is usually difficult. Pelvic and intestinal adhesions should be separated to restore the normal anatomic position of the ovary so as to avoid injury of ureter and pelvic vessels. Previous studies have shown that LESS is extremely limited in patients with severe pelvic adhesions, and such surgery is not recommended for these patients [14]. However, this study used JEON HG and other surgical concepts [9], also completed LESS for ovarian endometrioid cyst stripping surgery successfully, surgery is smooth, patients were well recovered. Therefore, it is feasible to use LESS to remove the endometrioid cyst under the condition of selecting suitable cases after the improvement of surgical skills and the accumulation of surgical experiences.

At present, the concept of minimally invasive surgery has been advocated by patients and surgeons, and widely used in a variety of obstetrics and gynecology surgery [15]. The advantages of laparoscopy include less trauma, rapid recovery, low recurrence rate and less pain, and LESS surgery is more minimally invasive than traditional laparoscopy surgery and more in line with cosmetic requirements, so it is increasingly favored by patients.

\section{Conclusions}

This study confirmed that laparoendoscopic single-site surgery for ovarian endometrioid cyst should be safe and feasible, but LESS for ovarian endometrioid cyst surgery is relatively difficult, so we should master the indications of surgery. It is believed that with the the advancement of endoscopic instruments and the improvement of operative skills, LESS will be prevalent and applied more and more widely in clinic.

\section{Funding}

This work was supported by grants from the Scientific Research Support Program for Postdoctoral of Jiangsu Province (2019 K064), the Major Science and Technology Program of Changzhou Health and Family Planning Commission (ZD201812), the Scientific Research Support Program for" 333 Project" of Jiangsu Province (Bra2019161). 


\section{References}

[1] F. P. Minson, M. S. Abrão, J. Sardá Júnior, D. C. Kraychete, S. Podgaec, F. D. Assis, [Importance of quality of life assessment in patients with endometriosis], Revista brasileira de ginecologia e obstetricia: revista da Federacao Brasileira das Sociedades de Ginecologia e Obstetricia 34 (1) (2012) 11-5.

[2] H. Kitchen, C. Seitz, A. Trigg, N. Aldhouse, T. Willgoss, H. Schmitz, A. Gater, C. Gerlinger, C. Haberland, Patients' and clinicians' perspectives on item importance, scoring, and clinically meaningful differences for the Endometriosis Symptom Diary (ESD) and Endometriosis Impact Scale (EIS), Health and quality of life outcomes 19 (1) (2021) 7.

[3] C. Peng, Y. Huang, Y. Zhou, Dydrogesterone in the treatment of endometriosis: evidence mapping and meta-analysis, Archives of gynecology and obstetrics (2021).

[4] A. N. Fader, L. Rojas-Espaillat, O. Ibeanu, F. C. Grumbine, P. F. Escobar, Laparoendoscopic single-site surgery (LESS) in gynecology: a multi-institutional evaluation, American journal of obstetrics and gynecology 203 (5) (2010) 501. e1-6.

[5] L. S. Bradford, D. M. Boruta, Laparoendoscopic single-site surgery in gynecology: a review of the literature, tools, and techniques, Obstetrical \& gynecological survey 68 (4) (2013) 295-304.

[6] X. Su, X. Jin, C. Wen, Q. Xu, C. Cai, Z. Zhong, X. Tang, Outcome of Gynecologic Laparoendoscopic Single-Site Surgery with a Homemade Device and Conventional Laparoscopic Instruments in a Chinese Teaching Hospital, BioMed research international 2020 (2020) 5373927.

[7] Y. J. Cho, M. L. Kim, S. Y. Lee, H. S. Lee, J. M. Kim, K. Y. Joo, Laparoendoscopic single-site surgery (LESS) versus conventional laparoscopic surgery for adnexal preservation: a randomized controlled study, International journal of women's health 4 (2012) 85-91.
[8] I. A. Al-Badawi, O. AlOmar, N. Albadawi, A. Abu-Zaid, Single-port laparoscopic surgery for benign salpingo-ovarian pathology: a single-center experience from Saudi Arabia, Annals of Saudi medicine 36 (1) (2016) 64-9.

[9] H. G. Jeon, W. Jeong, C. K. Oh, E. I. Lorenzo, W. S. Ham, K. H. Rha, W. K. Han, Initial experience with 50 laparoendoscopic single site surgeries using a homemade, single port device at a single center, The Journal of urology 183 (5) (2010) 1866-71.

[10] C. Hoyer-Sørensen, I. Vistad, K. Ballard, Is single-port laparoscopy for benign adnexal disease less painful than conventional laparoscopy? A single-center randomized controlled trial, Fertility and sterility 98 (4) (2012) 973-9.

[11] F. P. Buckley, 3rd, H. Vassaur, S. Monsivais, D. Jupiter, R. Watson, J. Eckford, Single-incision laparoscopic appendectomy versus traditional three-port laparoscopic appendectomy: an analysis of outcomes at a single institution, Surgical endoscopy 28 (2) (2014) 626-30.

[12] E. M. Pontarelli, C. Emami, N. X. Nguyen, M. Torres, D. M. Anselmo, Single-incision laparoscopic resection of ovarian masses in children: a preliminary report, Pediatric surgery international 29 (7) (2013) 715-8.

[13] G. O. Chong, D. G. Hong, Y. S. Lee, Single-port (OctoPort) assisted extracorporeal ovarian cystectomy for the treatment of large ovarian cysts: compare to conventional laparoscopy and laparotomy, Journal of minimally invasive gynecology 22 (1) (2015) 45-9.

[14] M. H. Chew, M. H. Chang, W. S. Tan, M. T. Wong, C. L. Tang, Conventional laparoscopic versus single-incision laparoscopic right hemicolectomy: a case cohort comparison of short-term outcomes in 144 consecutive cases, Surgical endoscopy 27 (2) (2013) 471-7.

[15] Y. Karasu, B. Akselim, D. Kavak Cömert, Y. Ergün, K. Ülker, Comparison of single-incision and conventional laparoscopic surgery for benign adnexal masses, Minimally invasive therapy $\&$ allied technologies: MITAT: official journal of the Society for Minimally Invasive Therapy 26 (5) (2017) 278-283. 